Rabaska

Revue d'ethnologie de l'Amérique française

HAMEl, NATHALIE. La Collection Coverdale. La construction

d'un patrimoine national. Québec, Presses de l'Université Laval, "Chaire Fernand-Dumont sur la culture ", 2009, 390 p. ISBN 978-2-7637-8929-3

\title{
Yvan Chouinard
}

Volume 8, 2010

URI : https://id.erudit.org/iderudit/045278ar

DOI : https://doi.org/10.7202/045278ar

Aller au sommaire du numéro

Éditeur(s)

Société québécoise d'ethnologie

ISSN

1703-7433 (imprimé)

1916-7350 (numérique)

Découvrir la revue

Citer ce compte rendu

Chouinard, Y. (2010). Compte rendu de [HAmel, NATHALIE. La Collection Coverdale. La construction d'un patrimoine national. Québec, Presses de

l'Université Laval, «Chaire Fernand-Dumont sur la culture », 2009, 390 p. ISBN

978-2-7637-8929-3]. Rabaska, 8, 203-206. https://doi.org/10.7202/045278ar d'utilisation que vous pouvez consulter en ligne.

https://apropos.erudit.org/fr/usagers/politique-dutilisation/ 
autochtone de fabrication de canards de bois, et finalement à l'émergence de cet art unique dont l'auteur est un représentant reconnu et estimé.

Assez curieusement, Fernand Gosselin, dans son essai, et François St-Onge, dans son anthologie, m'auront renvoyé malgré eux à la grande tradition du livre québécois. De tous les éditeurs québécois que j'aurai côtoyés et admirés - le père Paul-Émile Martin de Fides, Jacques Fortin de QuébecAmérique, Antoine del Busso, aujourd'hui aux Presses de l'Université de Montréal, Pascal Assasthyany de Boréal, Hervé Foulon chez HMH, le grand Pierre L'Espérance des Éditions de l'Homme -, je ne peux m'empêcher d'évoquer ici Denis Vaugeois. Il restera pour moi la figure éponyme de l'éditeur québécois, intelligent et brillant, charmeur et très à ses affaires. Mais en même temps un éditeur remarquable qui aura eu le courage de bâtir un fonds d'édition exceptionnel lié principalement à l'histoire en général et plus récemment à la question autochtone, dont il aura exploré pour nous le précieux héritage au profit de la société québécoise. Serge Lambert, éditeur de GID, m'apparaît de cette lignée.

RENÉ BOUCHARD

Ministère de la Culture, des communications et de la condition féminine, Québec

Hamel, Nathalie. La Collection Coverdale. La construction d'un patrimoine national. Québec, Presses de 1'Université Laval, "Chaire FernandDumont sur la culture », 2009, 390 p. ISBN 978-2-7637-8929-3.

Issu d'importants travaux de recherche pour l'obtention d'un diplôme de troisième cycle en ethnologie, cet ouvrage présente la fascinante aventure d'une collection d'objets devenue quasi mythique dans l'univers ethnohistorique et muséologique canadien. En voici les grandes lignes. Cette collection est celle d'une compagnie de navigation formée en 1912, la Canada Steamship Lines (CsL), qui possède aussi le Manoir Richelieu et l'Hôtel Tadoussac sur les rives du Saint-Laurent. En saison, les paquebots de la CsL desservent ces hôtels, à la plus grande joie des nombreux croisiéristes.

En 1922, le consultant en gestion d'entreprises William H. Coverdale (1871-1949), dont les bureaux sont à New-York, accepte l'offre de la CsL d'occuper la présidence de l'entreprise afin d'y implanter lui-même les recommandations de l'étude sur la situation financière de la compagnie qu'il vient de soumettre. L'auteur ne le clarifie pas, mais on suppose que n'y figure pas la recommandation de monter des collections, car ce n'est que plusieurs années plus tard que W. H. Coverdale se met à organiser, dans et autour des 
deux hôtels de la compagnie, d'impressionnants ensembles d'art graphique à thématique canadienne (Richelieu, à compter de 1928) et d'objets ethnographiques d'origine euroquébécoise et amérindienne (Tadoussac, à compter de 1940). Ces ensembles, en plus d'ajouter un caractère culturel très particulier aux qualités déjà fortes des lieux impliqués, sont destinés à augmenter l'attrait et la rétention des visiteurs. La richesse de ces collections est donc très utilisée par la suite dans les campagnes publicitaires vantant ces lieux de villégiature et aussi dans le but de promouvoir et de rehausser l'image corporative de la CSL.

Une quinzaine d'années après le décès du président Coverdale, la CsL abandonne son service de croisières et se départit ensuite des hôtels. Les multiples catalogues publiés ainsi que les expositions itinérantes réalisées au fil des ans font, entre autres, que la notoriété de la collection Coverdale n'est plus à faire. Des pressions populaires vont donc inciter les gouvernements québécois et canadien, après en avoir fait confirmer la valeur intrinsèque par ses spécialistes, à en acquérir l'essentiel. Ainsi, en 1968, le Musée du Québec hérite de la plupart des pièces de Tadoussac tandis que, quelques années plus tard, la majorité des œuvres du Manoir Richelieu seront partagées entre les Archives publiques du Canada (2 600 œuvres) et la Galerie Nationale du Canada (62 œuvres). Aujourd'hui, les mêmes institutions fédérales ont encore leurs pièces tandis que celles de Tadoussac ont été réparties entre le Laboratoire et la Réserve d'archéologie du Québec (2 600 artefacts), le Musée de la civilisation (3 300 objets) et le Musée national des beaux-arts (316 œuvres).

La relation de cette histoire demandait une recherche étendue et minutieuse et, sauf pour quelques aspects moins achevés, le résultat d'ensemble indique que Nathalie Hamel s'y est appliquée de manière assez rigoureuse. Cependant, thèse de doctorat oblige, le questionnement de l'auteur se devait de couvrir un horizon plus large qu'un simple récit historique. Ainsi, elle dira d'entrée de jeu que le point de départ de sa recherche était le suivant : «Pourquoi et comment cette collection d'entreprise a-t-elle pu prendre une telle importance dans les collections nationales et, donc, dans la formation d'un imaginaire collectif?» (p. 2). La question est justifiée et l'auteur commence par explorer les approches méthodologiques existantes afin de choisir une façon appropriée d'analyser les phénomènes pertinents. Donc, après avoir présenté les avantages de l'avenue conceptuelle de la « biographie culturelle » d'I. Kopytoff, elle dit pencher également pour l'approche practice, poetic et politic de Susan M. Pearce : « Cette approche permet d'aborder le collectionnement sous ses multiples facettes, tant ses aspects concrets que ses rôles sociaux, et elle permet d'en dégager une vision d'ensemble. Elle servira donc de trame de fond à notre analyse» (p. 24). En conclusion 
toutefois, c'est plutôt la structure de Kopytoff qui sert à parachever l'analyse (p. 324 à 327) : « Bref, ce parcours biographique a fait ressortir l'importance de la fabrication des valeurs par le collectionneur, l'acceptation de ces valeurs par les spécialistes du patrimoine, le rôle des actes officiels constitutifs du patrimoine comme aboutissement ou point de départ à une appropriation collective et, enfin, la place majeure qu'occupent la menace d'une perte et la perte effective dans la constitution du patrimoine. »

De fait, le grand responsable de l'élaboration de la collection de la CsL, W. H. Coverdale, a réussi à construire des ensembles qui parurent suffisamment « signifiants pour la société » que les spécialistes du patrimoine ont plus tard convenu de leur importance intrinsèque et aussi de leur signification identitaire toute spéciale (dans le contexte particulier de la décennie 1960-1970 faut-il préciser) pour ladite société et ont su, en même temps que certaines pressions populaires, convaincre les autorités politiques du moment de l'importance d'en faire l'acquisition pour enrichir les collections nationales québécoises et canadiennes, dans un contexte de crainte généralisé de voir dispersés des ensembles uniques.

L'étude de Nathalie Hamel se divise en deux grandes sections, « La construction d'un patrimoine » et "La transmission de l'héritage », qui correspondent respectivement à l'effort de collectionnement de W. H. Coverdale et à l'évolution ultérieure de la collection jusqu'à son intégration aux collections nationales. Précisons que, dans la première partie, en plus des œuvres d'art du Manoir Richelieu et des objets ethnographiques « canadiens-français » de l'Hôtel Tadoussac, on retrouve deux autres chapitres reliés à la collection. Le premier porte sur l'ensemble amérindien regroupé, sous la gouverne de W. H. Coverdale, dans un bâtiment se voulant la reconstitution, à proximité de l'hôtel, du probable établissement de traite initial entre Français et Amérindiens, le poste Chauvin érigé aux frais de la CsL selon des informations tirées des écrits de Champlain. Le second traite des boiseries de la maison Fargue/Estèbe de Québec que W. H. Coverdale a fait acheter en 1946 afin d'orner un prochain hôtel de la CsL. Quant à la seconde partie du livre, elle retrace les étapes de la destinée de la collection après le décès de $\mathrm{W}$. $\mathrm{H}$. Coverdale et son rôle dans la construction identitaire québécoise et canadienne. On y fait également mention de la lutte épique des gens de Tadoussac qui désirent rapatrier les éléments de la collection de l'hôtel et du poste Chauvin qui ont été déménagés à Québec. Le document s'achève sur cinq annexes et une bibliographie de 25 pages.

Un élément particulièrement utile à la compréhension, dans les annexes, est cette "Chronologie des événements relatifs à la collection Coverdale » (p. 351-364), qui met en parallèle depuis 1868 des événements liés à la collection elle-même et des événements liés à la conservation du patrimoine, 
au Québec, au Canada et aux États-Unis. On aurait aussi grandement apprécié une présentation sommaire, regroupée et éventuellement indexée des nombreux personnages auxquels on fait référence au fil de l'ouvrage. Un tel ajout aurait contribué à la compréhension d'ensemble de l'histoire de la collection. De même, il aurait probablement pu être éclairant d'ajouter, en annexe, le texte de la conférence donnée en octobre 1942 par William H. Coverdale à l'occasion d'une exposition regroupant 173 œuvres de la collection du Manoir Richelieu et « dans laquelle il raconte l'origine de la collection et son contenu » (p. 66).

Par ailleurs, afin d'enrichir son enquête et de mieux répondre à sa question de départ, l'auteur aurait aussi pu suivre la piste du Musée du Québec qu'elle soulève d'ailleurs (p. 7-8) en disant que la Commission des monuments historiques en réclamait la création dès 1923 afin de préserver «quelques spécimens de chacun des objets dont se servaient nos ancêtres ». Si ce musée, inauguré en 1933, avait collectionné jusqu'en 1965 dans le sens souhaité par la Commission, la collection Coverdale aurait-elle semblé aussi incontournable aux responsables de ce musée qui en recommandent alors l'acquisition ? A-t-il collectionné ? A-t-il mal collectionné ? Lui a-t-on donné les moyens de collectionner?

On regrette également de n'en pas apprendre davantage sur l'impact réel du président Coverdale dans la réussite globale de la compagnie et surtout sur la place des hôtels et de leurs collections, en terme de retombées économiques, dans l'ensemble des affaires de la compagnie. Un des seuls passages de l'étude s'approchant de cette problématique se trouve en page 118 , alors que W. H. Coverdale révèle un indice « montrant que la compagnie peut imposer certaines limites à ses activités de collectionnement » dans cette lettre qu'il adresse à un vendeur : " With such a large collection [...] as we have assembled, it would, of course, be difficult to pay very high prices for any particular object as we would soon run out of money and our stockholders would complain of lack of dividends ». L'auteur conclut ensuite par une question qui nous semble ambiguë et qui n'aura pas de suite : «Certains actionnaires auraient-ils réellement remis en cause la pertinence de ces investissements?»

Malgré ces lacunes et malgré certaines digressions et parenthèses dont l'utilité semble contestable à l'occasion, l'ouvrage réussit quand même, dans l'ensemble, à clarifier les principaux tenants et aboutissants de la notoriété de la collection Coverdale.

Yvan Chouinard

Société québécoise d'ethnologie 